\title{
Análisis de Sensibilidad aplicado a modelos de crecimiento urbano basados en autómatas celulares de estructura irregular
}

\author{
Urgilez-Clavijo Andrea ${ }^{1}$ | Gómez-Delgado Montserrat² | Barreira-González Pablo ${ }^{3}$ \\ Recibido: 30/07/2018 | Aceptado: 01/03/2019
}

\section{Resumen}

En el presente trabajo se aplica un Análisis de Sensibilidad (AS), como parte de un proceso de validación, sobre un modelo de simulación del crecimiento urbano basado en Autómatas Celulares (AC) de estructura irregular (MUGICA, Model for Urban Growth simulation using an Irregular Celular Automata). Este modelo ha sido desarrollado para simular el crecimiento urbano de tres municipios pertenecientes a un importante corredor urbano-industrial (Corredor del Henares) localizado en la zona central peninsular de España. Si bien la metodología utilizada en este trabajo ya ha sido aplicada en modelos basados en una estructura raster, se pretende comprobar su viabilidad en la fase previa de simulación, es decir, en el período de calibración (2000-2010) de un modelo de estructura irregular como MUGICA, el cual emplea la parcela catastral como unidad de referencia. El objetivo es explorar el grado de influencia de cada uno de los factores en los resultados del modelo, de manera individual y en sus diferentes combinaciones. Para ello se realiza una eliminación sucesiva de los factores con el fin de evaluar si la ausencia de uno o varios de ellos supone una alteración significativa de los resultados. Los resultados muestran, en primer lugar, la viabilidad de la aplicación de esta metodología en este tipo de entorno irregular. Por otro lado, se ha podido constatar la gran influencia de los factores accesibilidad y aptitud en el desarrollo de suelo urbano, como sería de esperar en un modelo de estas características.

Palabras clave: Análisis de sensibilidad; autómatas celulares irregulares; simulación del crecimiento urbano

\section{Abstract \\ Sensitivity Analysis applied on an urban growth cellular automata based-model with irregular structure}

This work presents a Sensitivity Analysis (SA), as part of a validation process that is applied on an urban growth Cellular Automata (CA) based-model with irregular structure (MUGICA, Model for Urban Growth simulation using an Irregular Cellular Automata). This model has been developed to simulate urban growth of three municipalities located in an important industrial corridor (Corredor del Henares) in the central area of Spain. Although the methodology employed in this work has already been applied in models based on a raster structure, it is intended to verify its viability in the previous simulation phase, that is, in the calibration period (2000-2010) in a model

\footnotetext{
1. Universidad del Azuay, Instituto de Estudios de Régimen Seccional del Ecuador. Universidad de Zaragoza, Departamento de Geografía y Ordenación del Territorio. aurgilez@uazuay.edu.ec

2. Universidad de Alcalá, Departamento de Geología, Geografia y Medio Ambiente. montserrat.gomez@uah.es

3. Universidad de Alcalá, Departamento de Geología, Geografia y Medio Ambiente. pablobarreiragonzalez@gmail.com
} 
of irregular structure such as MUGICA, which uses the cadastral plot as reference unit. This procedure aims to explore the degree of influence of each of the parameters on the results of the model, individually and as a whole. For this purpose, a successive elimination of the parameters is performed to evaluate if the absence of one or several of them implies a significant alteration of the results. The results show, firstly, the viability to apply this methodology in a vector environment. On the other hand, it has been possible to verify the significative influence of the suitability and accessibility factors in the development of urban land, as would be expected in a model of these characteristics.

Keywords: Sensitivity analysis; irregular cellular automata; urban growth simulation

\section{Résumé}

\section{Analyse de Sensibilité d’un modèle de simulation de croissance urbaine basée sur les automates cellulaires avec structure irrégulière}

Dans cet article, on utilise l'analyse de sensibilité (AS), dans le cadre d'un processus de validation d'un modèle de simulation de croissance urbaine. Celle-ci est basée sur les Automatas Cellulaires (CA) sur une structure irrégulière nommée le modèle (MUGICA, Model for Urban Growth simulation using an Irregular Cellular Automata). Ce modèle a été développé pour simuler la croissance urbaine de trois municipalités appartenant à un important corridor urbain-industriel (Corredor del Henares) situé dans la zone péninsulaire centrale de l'Espagne. Bien que la méthodologie utilisée dans ce travail ait déjà été appliquée dans des modèles basés sur une structure matricielle, elle est destinée à vérifier sa viabilité lors de la phase de simulation précédente, c'està-dire pendant la période d'étalonnage (2000-2010) dans un modèle de structure irrégulière telle que MUGICA, qui utilise le tracé cadastral comme unité de référence. Lobjectif est d'explorer le degré d'influence de chacun des facteurs dans les résultats du modèle, individuellement et selon différentes combinaisons. Pour cela, une élimination successive des facteurs a été effectuée afin d'évaluer si l'absence d'un ou plusieurs d'entre eux suppose une altération significative des résultats. Les résultats montrent, en premier lieu, la viabilité de l'application de cette méthodologie dans ce type d'environnement irrégulier. En second lieu, il a été possible de confirmer la grande influence des facteurs d'accessibilité et d'aptitude dans le développement des terrains urbains. Comme on pouvait s'y attendre dans un modèle présentant ces caractéristiques.

Mot clés: analyse de sensibilité; automatas cellulaires irréguliers; simulation de croissance urbaine

\section{Introducción}

La dinámica de los cambios de uso de suelo, en especial el crecimiento urbano, ha conllevado importantes implicaciones ambientales y socio-territoriales, provocadas por diferentes actividades antrópicas (Kalnay y Cai, 2003; Antrop, 2004; Salvati y Sabbi, 2011) y caracterizadas principalmente por la pérdida de recursos y del terreno natural, trayendo consigo consecuencias no deseables tanto sociales como económicas y políticas (Barredo et al., 2004) o de degradación del paisaje (Verburg et al., 2004). Si bien las grandes zonas pobladas son una clara representación de las actividades antrópicas (de Noronha y Vaz, 2015; Saraiva y Pinho, 2017), también lo son las áreas periféricas a las grandes ciudades, que han venido desarrollando de forma exacerbada el crecimiento del uso urbano y acentuándose las presiones sobre la zona periurbana y corredores de acceso a las grandes ciudades (Antrop, 2004; Muller et al., 2010; Fahmi et al., 2014). 
Ante esta situación, la comunidad científica ha centrado sus esfuerzos en desarrollar herramientas que permitan la simulación del fenómeno de crecimiento urbano, con el objetivo de entender fácilmente los procesos y factores derivados de éste (Verburg et al., 2004). Además, a través de estas herramientas, es posible reproducir dinámicas y procesos pasados (Paegelow y Camacho, 2008), así como su evolución en el futuro (Hansen, 2010; Santé et al., 2010). Entre otros, las técnicas de evaluación multicriterio, los modelos basados en agentes y los modelos basados en Autómatas Celulares (AC) han sido utilizados para simular este tipo de fenómenos, siendo éstos últimos ampliamente empleados dada su simplicidad, flexibilidad, transparencia y su capacidad para ser integrados con los Sistemas de Información Geográfica (SIG) (Santé et al., 2010; Triantakonstantis y Mountrakis, 2012).

Los AC modelan sistemas dinámicos y complejos que evolucionan en lapsos temporales discretos, en los que las interacciones locales entre sus componentes generan cambios globales en el espacio. Su aplicación en el modelamiento de las dinámicas urbanas partió de modelos teóricos que simulaban estructuras urbanas simples (Wolfram, 1984). Posteriormente se desarrollaron varios estudios (Batty y Xie, 1994; Batty y Xie, 1997; Semboloni, 1997; Batty et al., 1999) que permitieron probar los diferentes enfoques teóricos mediante la simulación de formas urbanas generales. Con la aparición de los SIG se rompió la brecha entre las aplicaciones artificiales y la simulación del mundo real, brindándonos la posibilidad de aplicar los anteriores modelos teóricos en la simulación de procesos de desarrollo urbano reales y extender su aplicabilidad a diferentes ámbitos de estudio (Batty y Xie, 1994; Engelen et al., 1999; Barredo et al., 2004; Kocabas y Dragicevic, 2006; Stevens y Dragicevic, 2007; Aguilera et al., 2010; Pinto y Antunes, 2010; Dahal y Chow, 2014; Berberoglu et al., 2016, Cao et al., 2016, Liu et al., 2016).

La utilidad de los modelos basados en autómatas celulares en la simulación prospectiva del crecimiento urbano ha sido demostrada desde hace ya varias décadas, y así lo atestigua el importante volumen de publicaciones que podemos encontrar actualmente en la literatura científica (Batty et al., 1999; Barredo et al., 2004; Paegelow y Camacho, 2008; Hansen, 2010; Santé et al., 2010, entre otros). De hecho, en un reciente trabajo de revisión sobre modelos aplicados en este campo, se confirmaba que más del $80 \%$ de los trabajos publicados utilizaban para este fin este tipo de modelos, por encontrarse ya su eficiencia suficientemente contrastada y probada (Triantakonstantis y Mountrakis, 2012).

El proceso de calibración y validación de los modelos son, en conjunto, los procesos que permiten evaluar la eficacia de las simulaciones (van Vliet et al., 2016). Una previa calibración es fundamental para reproducir un evento de desarrollo urbano al ser comparado con el evento real. Tal hecho garantiza en mayor medida la exactitud del modelo. Por otro lado, en la calibración se mide de manera directa la capacidad de reproducir un evento de desarrollo urbano presente, basado en información del pasado y realizando una contrastación con datos reales. Adicionalmente a la calibración, es importante aplicar algún tipo de proceso de validación que permita evaluar la correcta implementación del modelo y la utilización del mismo para simular el fenómeno tratado a futuro, cuyos resultados no es posible contrastar con datos reales (Engelen y White, 2008). Uno de estos procedimientos de validación consiste en la aplicación de un análisis de sensibilidad (AS), que permite medir la variabilidad de los resultados generados con respecto a los cambios efectuados sobre los factores de entrada, comprobando así la influencia de cada uno de estos factores sobre el comportamiento del modelo (Kocabas y Dragicevic, 2006; Xu y Zhang, 2013; Hewitt y Díaz Pacheco, 2017). Sin embargo, como se recoge en el trabajo de Van Vliet et al. 
(2016), los AS continúan sin aplicarse de forma habitual en los modelos de cambios de usos del suelo, a pesar de ser especialmente apropiados por incluir cierto componente de estocasticidad.

Existen ya diferentes trabajos en los que se estudia la influencia de los factores de un modelo sobre los resultados generados utilizando el enfoque de Análisis de Sensibilidad Global o GSA por sus siglas en inglés (Global Sensitivity Analysis) (Crosetto y Tarantola, 2001; Gómez-Delgado y Tarantola, 2006; Lilburne y Tarantola, 2009; Plata-Rocha et al., 2012,). Barreira-González et al. (2015a) propone una metodología complementaria a este AS en la que se determina la influencia de cada factor que compone el modelo en los resultados obtenidos mediante el cálculo de los efectos principales y totales. Dicho estudio se realizó en un modelo de simulación de crecimiento urbano basado en autómatas celulares de estructura regular (raster).

En el contexto de los modelos basados en AC que emplean estructuras irregulares para representar el terreno (Stevens et al., 2006; Moreno et al., 2008; Dahal y Chow, 2015; Lu et. al, 2015; Abolhasani et al., 2016), el número de trabajos hasta la fecha es sumamente inferior en comparación con los que emplean estructuras regulares, siendo testimonial la existencia de trabajos que en este contexto apliquen algún proceso de validación, en general, y de AS, en particular. Dahal y Chow (2015) realizan un estudio de la sensibilidad de su modelo basado en estructuras irregulares a variaciones en la configuración de la vecindad. Por su parte, Abolhasani et al. (2016) también estudian el efecto de la configuración de la vecindad en la implementación de diferentes escenarios de simulación del crecimiento urbano a partir del modelo ParCA (que emplea parcelas catastrales para representar el territorio) a través de indicadores de compacidad, compatibilidad y dependencia.

Dada la escasez por tanto de estudios en los que se realice un AS en modelos basados en estructuras irregulares, y que tenga en cuenta todos los factores del modelo, el objetivo del presente trabajo es comprobar la viabilidad de la utilización del AS propuesto por Barreira-González et al., (2015a), a un modelo de simulación de crecimiento urbano basado en autómatas celulares irregulares (MUGICA, Model for Urban Growth Simulation Using an Irregular Cellular Automata). Este modelo fue aplicado en tres municipios del Corredor del Henares, pertenecientes a las Comunidades Autónomas de Madrid y Castilla La-Mancha. Realizada una primera aproximación en un trabajo anterior (Urgilez et al., 2018), el presente estudio presenta una metodología más completa y sistemática, con un análisis más pormenorizado de los resultados.

Este AS, además, se aplica de manera novedosa al periodo de calibración del modelo (20002010), dada la importancia de conocer la influencia de cada factor en los resultados durante este periodo, ya que es en éste en el que se realizan todos los posibles ajustes de los factores que serán empleados en el proceso de validación de la simulación a futuro, para lo que no se van a disponer, en ningún caso, de datos reales con los que contrastar los resultados (Barreira-González et al., 2015a). Esta inquietud e investigación surge de los resultados del trabajo citado, en el que se pudo comprobar mediante la aplicación de este AS que, a pesar de haber sido calibrado el modelo correctamente, cuando se utilizaba para realizar simulaciones a futuro, algunos de los factores como la accesibilidad no estaban teniendo en los resultados del modelo la importancia y efecto que se les presuponía.

Este trabajo comienza con la descripción del área de estudio y el modelo de partida (epígrafes 2 y 3), posteriormente se presenta la metodología utilizada para determinar la influencia de cada uno de los factores mediante el cálculo de porcentaje de acuerdo, efectos principales y totales, y 
mapas de frecuencias (epígrafe 4). Finalmente se presentan en conjunto los resultados (epígrafe 5), así como la discusión y conclusiones obtenidas del trabajo (epígrafe 6).

\section{2. Área de estudio}

El área de estudio está conformada por los municipios de Los Santos de la Humosa, Meco y Azuqueca de Henares, pertenecientes al Corredor del Henares, una importante y dinámica área urbano-industrial que recorre dos Comunidades Autónomas diferentes: Madrid y Castilla LaMancha (Mapa 1). Este corredor se caracteriza por un crecimiento urbano longitudinal, desarrollado paralelamente al eje vial de la carretera A-2 que conecta Madrid y Guadalajara, a la vía de ferrocarril Madrid-Zaragoza y al río Henares. Las transformaciones experimentadas por este territorio entre los años 2000 y 2010 están en consonancia con lo sucedido en la región funcional de Madrid, una de las áreas en las que se ha experimentado una mayor transformación (tanto a nivel nacional como europeo) debido al desarrollo de nuevas zonas urbanas en las últimas dos décadas, (Plata Rocha et al., 2009; Hewitt y Escobar Martínez, 2011; Olazabal y Bellet, 2018). De hecho, esta zona ha sido ya objeto de varios estudios de simulación del crecimiento urbano con varias técnicas y en diferentes períodos, pero a una escala regional o empleando siempre como unidad espacial base el píxel (Barredo y Gómez-Delgado, 2008; Plata Rocha et al., 2011; DíazPacheco y Hewitt, 2014; Cantergiani y Gómez-Delgado, 2018).

Dentro de esta región funcional, este corredor se conforma como una zona estratégica a nivel nacional, concentrando funciones económicas importantes y siendo centro neurálgico de transporte (aquí se encuentra el principal aeropuerto del país: Madrid-Barajas Adolfo Suárez), empleo o administrativo, entre otros (Cantergiani y Gómez Delgado, 2016). De hecho, en la última década se está experimentando también un aumento y transformación muy importante del suelo productivo, siendo actualmente muy notable la presencia del sector logístico, actividad que requiere mucha más superficie para su desarrollo que las actividades que tradicionalmente ocupaban este área (sector farmacéutico, químico, sector blanco o de producción de electrodomésticos, entre otros).

Si bien el desarrollo en el eje central está ya muy consolidado, todavía hay zonas en el margen norte, y especialmente en el margen sur, que cuentan con un importante volumen de suelo urbanizable. Por lo tanto, esta área se presenta como un interesante laboratorio para realizar ejercicios de simulación de crecimiento urbano a futuro (tanto residencial como productivo) que permita realizar prácticas de planificación más informadas y sostenibles.

Como puede comprobarse en parte en el Mapa 1, la selección de los municipios se realizó de tal forma que hubiese diferencias significativas en cuanto a su localización respecto al eje de máxima accesibilidad (Azuqueca de Henares en el eje central, Meco en el margen Norte y Los Santos de la Humosa en el margen Sur), disponibilidad de suelo urbanizable, componente rural (mucho más acusada en el municipio madrileño de Los Santos de la Humosa), características socioeconómicas e incluso topográficas (los dos primeros se sitúan en el valle del Henares, mientras que el último se encuentra en el páramo, por encima de los $900 \mathrm{~m}$ ). Por otro lado, aunque las políticas de ordenación urbana en las últimas décadas han favorecido el aumento de área urbanizada y suelo urbanizable en toda el área, el aumento real ha sido diferente en cada uno de los municipios, pasando entre el año 2000 y 2010 de 29 a 42 ha en el municipio de Los Santos de la Humosa, de 98 a 318 ha en el municipio de Meco y de 322 ha a 576 en Azuqueca de Henares (Dirección General de Catastro, 2013). El aumento de población experimentado también ha sido diferente en los 
distintos municipios, pasando en estos mismos años de 19.491 a 33.735 habitantes en Azuqueca de Henares (34.768 en 2017), de 6.328 a 12.580 en Meco (13.570 en 2017) y de 906 a 2.165 en Los Santos de la Humosa (2.486 en 2017) (INE, 2017).

Mapa 1. Localización y delimitación del área de estudio.
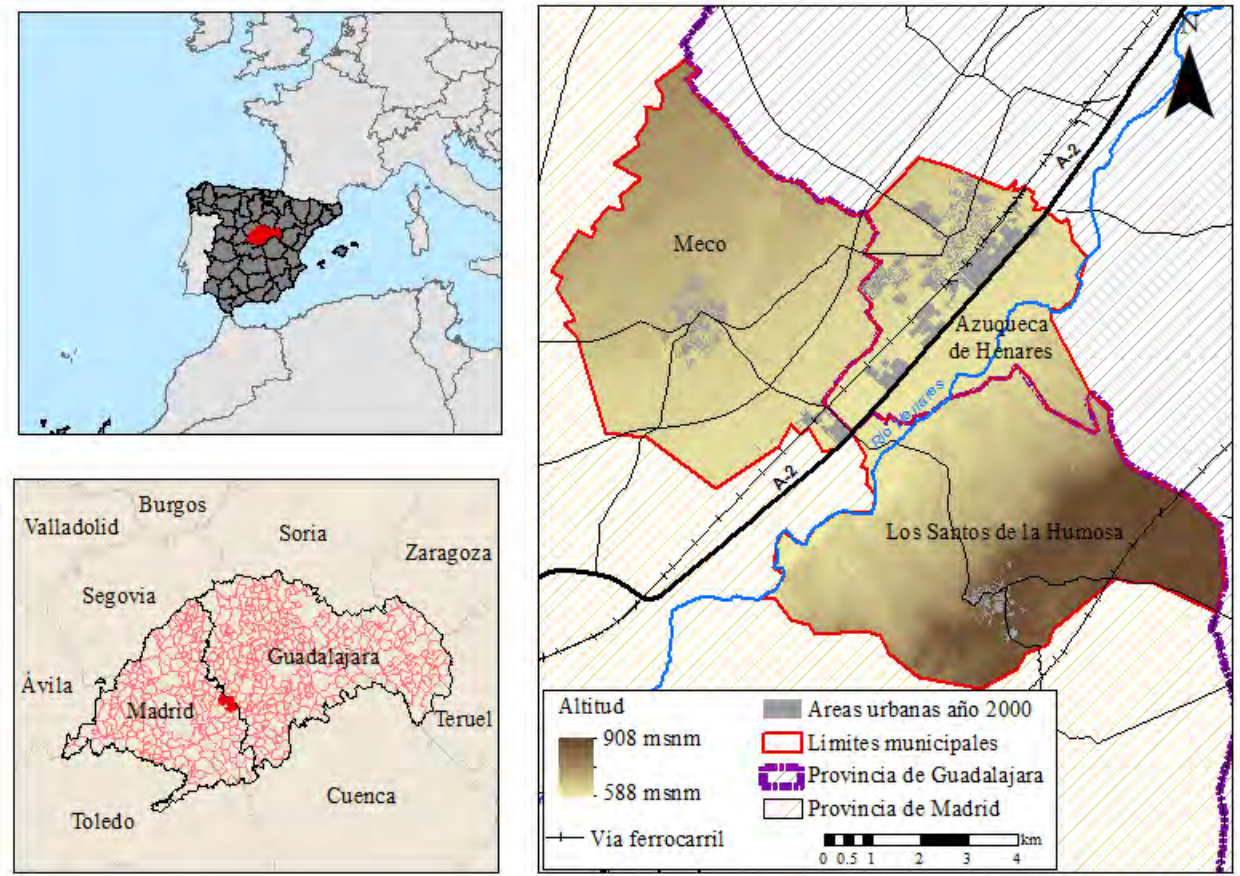

Fuente: elaboración propia a partir de datos del Instituto Geográfico Nacional (IGN, 2016).

\section{Modelo de partida}

El modelo desarrollado para la simulación del crecimiento urbano a futuro en esta zona de estudio (MUGICA) está basado en el tradicional esquema NASZ (Neigborhood, Accessibility, Suitability, Zoning Status) propuesto por White et al. (1997), que combina los factores de vecindad, accesibilidad, aptitud y zonificación, incluyendo además una componente de aleatoriedad, la cual otorga al modelo la capacidad de reproducir incertidumbre en el espacio y en el tiempo (BarreiraGonzález et al., 2015b; Barreira-González et al., 2017). A diferencia de los modelos tradicionales basados en autómatas celulares, cuya unidad espacial base es el píxel, este modelo utiliza estructuras espaciales irregulares, usando como fuente principal la base de datos de la Dirección General del Catastro. Está desarrollado en el lenguaje de programación Python e incluye librerías de código abierto y comercial como OGR, de Geospatial Data Abstraction Library (GDAL) (Open Source Geospatial Foundation, 2000) y Arcpy de ArcGIS, respectivamente.

La forma de representación del espacio utilizando una estructura irregular, y las operaciones asociadas a esta, implicaban un aumento sustancial en el tiempo computacional de ejecución del modelo. Para resolver este problema se aplicó la teoría de grafos, abstrayendo la representación vectorial a un grafo, donde cada parcela se representa como un nodo y los enlaces conectan parcelas consideradas vecinas, almacenándose en ambos toda la información de las distintas parcelas como el identificador de parcela, uso del suelo, año de desarrollo, aptitud de la parcela para su 
desarrollo como suelo urbano si todavía no está construida, valor de accesibilidad, estado de zonificación, parcelas vecinas, etc. (Barreira-González et al., 2015b).

Cada iteración del modelo representa un año natural, en el que las parcelas son clasificadas o no como suelo vacante. La condición de suelo vacante desarrolla un valor de potencial que puede ser residencial o productivo. Este potencial de transición a un nuevo uso urbano se calcula mediante:

$$
P_{i, k}=N_{i, k} A_{i} S_{i} Z_{i}+R
$$

[Ecuación 1]

Donde para cada parcela i, la transición $\mathrm{k}$ dependerá de la vecindad $(\mathrm{N})$, la accesibilidad (A), la aptitud (S), la zonificación legal (Z) y el factor estocástico o de aleatoriedad (R).

La vecindad en este modelo es la región parcial o totalmente cubierta por el buffer generado alrededor de cada parcela. El efecto vecindad se materializa en el modelo a través de funciones de atracción-repulsión previamente calculadas, considerando el uso del suelo urbano preexistente (Barreira González y Barros, 2016). En este caso se empleó una distancia de 250 m. (BarreiraGonzález et al., 2017).

Las áreas industriales y comerciales requieren conexiones eficientes mediante una red de transporte de alta capacidad, y las áreas residenciales y los centros urbanos requieren de múltiples vías de acceso, por lo que la accesibilidad no debería basarse sólo en la proximidad a la red de carreteras, sino también en la conectividad entre estas. Así, este factor se determinó a partir del cálculo de varias métricas para obtener un único valor basado en las menores distancias recorridas y tiempos óptimos empleados hasta la red de carreteras, seleccionando la métrica que mejor reprodujo el crecimiento urbano en el período de calibración. En este caso se empleó la distancia mínima recorrida para acceder desde cada parcela a los nodos principales de la red de transporte (Barreira-González et al., 2017). Por su parte, la aptitud o capacidad intrínseca de cada punto del territorio analizado para albergar un uso urbano, fue el resultado de la aplicación de una regresión logística espacial en la que se evaluaron los coeficientes para cada variable que explicaba el crecimiento urbano entre 1990 y 2000 (crecimiento de la población, uso del suelo, pendiente, suelo protegido, composición geológica, distancia a cuerpos de agua, entre otros) (Barreira-González et al., 2015a). La zonificación se definió en función de los Planes Generales de Ordenación Urbana vigentes. Finalmente, la aleatoriedad se introdujo como el parámetro que habitualmente se utiliza en este tipo de modelos para reproducir la incertidumbre asociada a los procesos en los que interviene el ser humano, pero en este caso se consideró una componente adicional, asociada al crecimiento urbano irregular. Esta componente denominada Random Protected Land Development (RPLD) pretende reproducir la aparición de asentamientos urbanos irregulares, no contemplados en los planes de ordenación, pero que se ha venido produciendo en ciertos municipios de la Comunidad de Madrid.

El modelo fue calibrado para el periodo 2000 - 2010, consiguiéndose un acuerdo del $61,7 \%$ con respecto al crecimiento observado para el periodo de calibración, un ajuste bastante elevado, considerando que se utilizó para el cálculo sólo la superficie residencial nueva (cambios experimentados en el período de estudio) y no toda la superficie residencial como se suele hacer en la calibración de este tipo de modelos. Como venimos mencionando, esta sería una breve descripción de las bases del modelo. Para una explicación más detallada de MUGICA y su funcionamiento consultar Barreira-González, et al. (2017). 


\section{Metodología}

La metodología para realizar la validación del modelo a partir de un AS, está basada en el planteamiento de Barreira-González, et al. (2015a) que a su vez es una adaptación de los métodos de Análisis de Sensibilidad Global (Global Sensitivity Analysis - GSA) y OAT por sus siglas en inglés (One-factor-At-a-Time) (Saltelli, et al., 2000) en la que se omite el uso de variaciones probabilísticas simultáneas en todos los factores de entrada y se asignan uno a uno los factores a evaluar. El GSA consiste en determinar qué factores del modelo están influyendo en la incertidumbre o variación de los resultados del mismo y, lo que resulta todavía más importante, en qué proporción. Para ello se calcula una serie de índices de sensibilidad que representan la contribución de cada factor de entrada (parámetro del modelo) en la varianza de la salida. La adaptación consiste en simplificar el método GSA, para generar diferentes simulaciones del modelo donde los factores sean introducidos de uno en uno (OAT) y así conformar la muestra estadística de análisis.

En la Imagen 1 se representa esquemáticamente la metodología utilizada para la evaluación de la influencia de los factores del modelo MUGICA para el periodo de calibración que se explicará en los siguientes apartados. Recordemos que, a diferencia de otras aplicaciones de este tipo de análisis de sensibilidad, aquí se realizará de manera novedosa durante el período de calibración, para fortalecer, por tanto, los resultados de este proceso y, por otro lado, dar mayor robustez a los resultados de las simulaciones que con este modelo se realicen a futuro y para lo que no disponemos de datos reales con los que realizar ningún tipo de validación.

Imagen 1. Esquema metodológico para la aplicación del AS al modelo MUGICA.

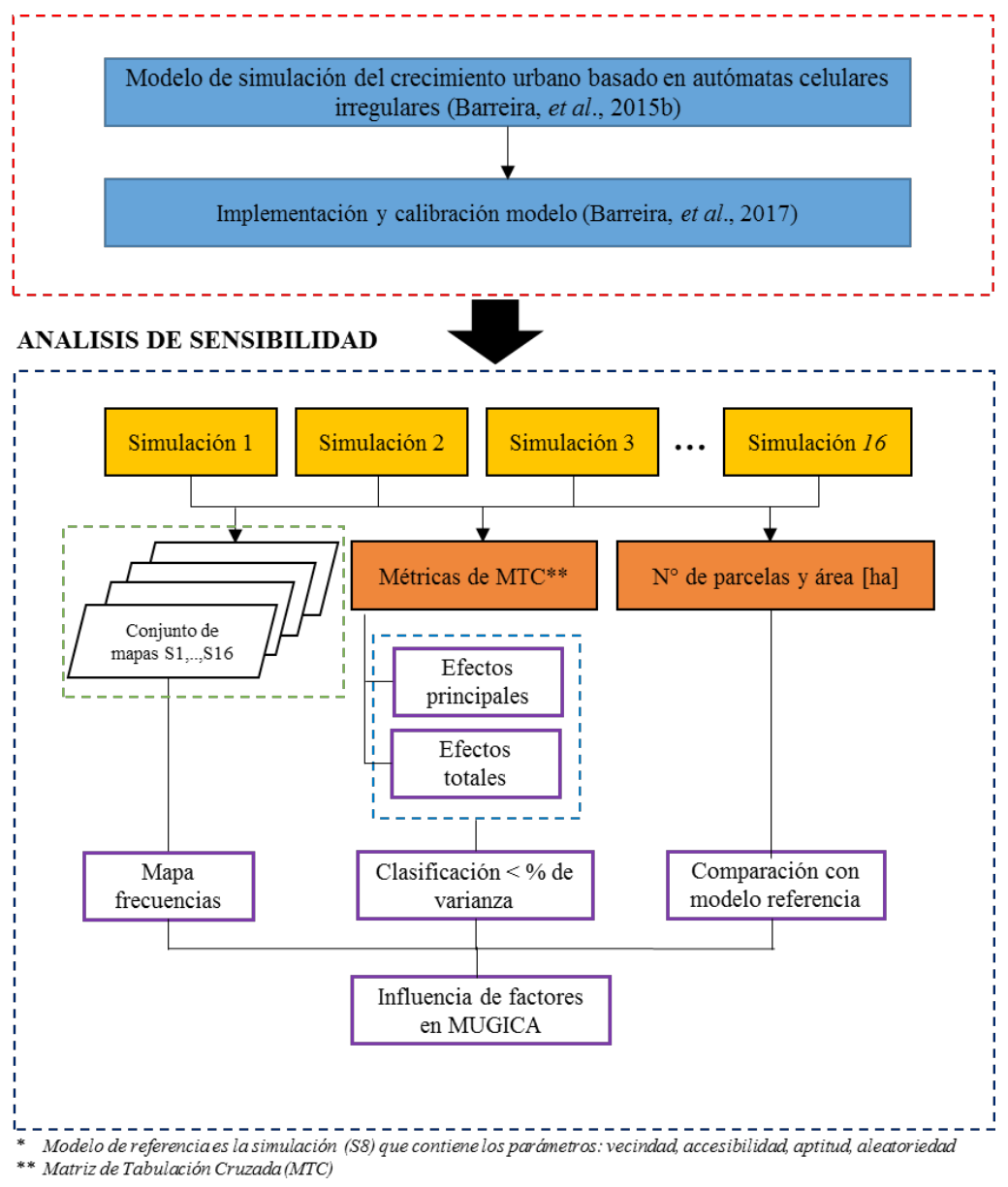


Se parte por tanto de un modelo con 4 factores ( 88 - modelo de referencia) y se generan todas las versiones adicionales del modelo posibles, combinando todos los factores excepto la zonificación, por la complejidad que generaría el análisis, ya que se trata, en realidad, de una restricción, no de un factor propiamente dicho. Por tanto, la zonificación se mantiene en cada una de las versiones adicionales del modelo. Por otro lado, es necesario incluir una versión del modelo que no incluya ninguno de los 4 factores (S16) para poder hacer la comparativa completa (Cuadro 1).

Cuadro 1. Modelos simulados de acuerdo con la combinación de factores que influyen en el desarrollo de potencial del suelo.

\begin{tabular}{|c|c|c|c|c|c|c|c|c|c|c|c|c|c|c|c|c|}
\hline \multirow{2}{*}{ Factores } & \multicolumn{16}{|c|}{ Simulaciones (S) } \\
\hline & S1 & $\mathrm{S} 2$ & S3 & S4 & S5 & S6 & S7 & S8 & S9 & S10 & S11 & S12 & S13 & S14 & S15 & S16 \\
\hline Accesibilidad (A) & $x$ & $x$ & & $x$ & & $x$ & & $x$ & & & $x$ & & $x$ & & $x$ & \\
\hline Aptitud (S) & $x$ & & $\mathrm{x}$ & $\mathrm{x}$ & & & $\mathrm{x}$ & $x$ & & & & $\mathrm{x}$ & $\mathrm{x}$ & $\mathrm{x}$ & & \\
\hline Vecindad (N) & $x$ & $x$ & $\mathrm{x}$ & & $\mathrm{x}$ & & & $\mathrm{x}$ & & $\mathrm{x}$ & & & & $\mathrm{x}$ & $\mathrm{x}$ & \\
\hline Aleatoriedad (R) & & & & & & & & $\mathrm{x}$ & $\mathrm{x}$ & $\mathrm{x}$ & $\mathrm{x}$ & $\mathrm{x}$ & $\mathrm{x}$ & $\mathrm{x}$ & $\mathrm{x}$ & \\
\hline
\end{tabular}

\subsection{Métricas obtenidas a partir de la Matriz de Tabulación Cruzada (MTC)}

En primer lugar, los resultados de desarrollo urbano de todos los modelos se comparan con el de referencia (S8) y se genera la correspondiente MTC. A diferencia de lo que ocurre con los modelos basados en celdas regulares, al trabajar con unidades espaciales irregulares habría dos opciones: trabajar con el número de parcelas desarrolladas o con el área de las parcelas desarrolladas. Obviamente lo más apropiado es trabajar con esta última, debido a las diferencias geométricas que pueden existir entre ellas y, por tanto, de superficie. Esto implica un cálculo adicional a lo que habitualmente se realizaría cuando se trabaja con celdas regulares, pero, por otro lado, se trata de un resultado más realista.

De esta MTC se derivan tres métricas basadas en la exactitud del productor para cada uno de los modelos (Cuadro 2): exactitud de la ubicación del crecimiento residencial (Residential Producer Accuracy - RPA), exactitud de la ubicación del crecimiento productivo (Productive Producer Accuracy - PPA) y exactitud de la ubicación del crecimiento urbano (Growth Location Accuracy - GLA) (Chuvieco, 2010; Barreira-González et al., 2017). Las dos primeras determinan la proporción de la simulación por uso de suelo localizado correctamente de acuerdo con el crecimiento del modelo de referencia, y la segunda evalúa la exactitud de la localización del crecimiento, independientemente del uso del suelo simulado. A partir de estas métricas se desarrollará el cálculo descrito en el siguiente apartado. 
Finalmente se calcula el efecto total de cada factor mediante la suma del efecto principal del factor y los efectos combinados de ese factor con el resto de los factores.

$$
\eta_{T i}=\eta_{i}+\sum_{\substack{j \neq i \\ \text { [Ecuación 5] }}}^{n} \eta_{i j}+\ldots+\eta_{i j \ldots n}
$$

En este caso, el cálculo está simplificado respecto al original (Saltelli et al., 2000), puesto que los resultados del modelo tienen una distribución discreta con solo dos valores ( 1,0 : urbanizado, no urbanizado), a diferencia de otras aplicaciones de este tipo de índices en el campo del modelado numérico.

\subsection{Mapa de frecuencias}

Para comparar el conjunto de los modelos simulados se utiliza un mapa de frecuencias, que representa el número de veces que una parcela ha desarrollado un uso urbano (residencial o productivo) en la combinación de factores definida por los 16 modelos generados (Cuadro 1). Este método es comúnmente utilizado para este tipo de análisis (Gómez-Delgado y Bosque Sendra, 2004; Brown et al., 2005; Barreira-González et al., 2015a) y ofrecen una información adicional y una visión rápida y clara de dónde se localizan las zonas más sensibles a las modificaciones realizadas en los factores de entrada del modelo.

La metodología propuesta genera resultados que permiten determinar las parcelas en las que recurrentemente se desarrolla un determinado uso en cada iteración, independientemente de los factores que estén afectando a cada modelo. Por lo tanto, permite identificar zonas prioritarias, es decir, menos sensibles a los cambios o incertidumbres introducidos en el modelo y, por tanto, más robustos, puesto que son los resultados más repetidos (Gómez-Delgado y Bosque Sendra, 2004).

\section{Resultados}

\subsection{Comparación de los resultados de las simulaciones}

$\mathrm{Al}$ analizar las métricas obtenidas de la MTC (Cuadro 3), se observa de forma general que las simulaciones S1, S4 y S15 para el desarrollo de suelo urbano son muy similares al modelo de referencia (S8), con valores en todas las métricas superiores al 83\%. Esto se corrobora en el Mapa 2, donde aparecen los resultados para ambos tipos de usos (residencial y productivo) en estas tres simulaciones (escogidas por ser las más representativas), acompañadas del modelo S8. Hemos de advertir que la simulación se realiza con los tres municipios en conjunto, sin embargo, para mejorar la visualización de los resultados, se presentan en el mapa de forma separada.

Por otro lado, podemos confirmar que la aleatoriedad, como era de esperar, tanto de forma individual como combinada con otros factores, es la que produce mayores variaciones respecto al modelo de referencia, arrojando las distintas métricas valores muy bajos. En el caso del suelo residencial, se observa cómo la utilización exclusiva de factores como la vecindad o la aptitud no consiguen por sí solos generar los mismos resultados que el modelo de referencia, al contrario que la accesibilidad (S6) que consigue un ajuste bastante elevado (89,2\%). 
Cuadro 3. Resultados del cálculo de las tres métricas obtenidas de la MTC generada en cada simulación del modelo (S1-S16).

\begin{tabular}{|c|c|c|c|}
\hline Modelos & RPA & PPA & GLA \\
\hline S1 (N, A, S) & 90,99 & 94,09 & 93,08 \\
\hline S2 (N, A) & 89,21 & 82,99 & 85,02 \\
\hline S3 (N, S) & 62,20 & 81,98 & 75,53 \\
\hline S4 (A, S) & 90,78 & 94,09 & 93,01 \\
\hline S5 (N) & 58,91 & 85,04 & 76,52 \\
\hline S6 (A) & 89,21 & 84,27 & 85,88 \\
\hline S7 (S) & 65,20 & 82,55 & 76,89 \\
\hline S9 (R) & 49,04 & 76,11 & 67,28 \\
\hline S10 (N, R) & 54,66 & 77,48 & 70,04 \\
\hline S11 (A, R) & 83,52 & 91,34 & 88,79 \\
\hline S12 (S, R) & 61,69 & 96,71 & 85,30 \\
\hline S13 (A, S, R) & 83,70 & 83,10 & 83,30 \\
\hline S14 (N, S, R) & 62,62 & 94,77 & 84,29 \\
\hline S15 (N, A, R) & 83,35 & 96,80 & 92,42 \\
\hline S16 & 61,34 & 84,74 & 77,11 \\
\hline
\end{tabular}

RPA: Residential Producer Accuracy; PPA: Productive Producer Accuracy; GLA: Growth Location Accuracy $\mathrm{N}$ : Vecindad; A: Accesibilidad; S: Aptitud; R: Aleatoriedad

Observando ambos resultados (métricas y localización espacial), comprobamos que las simulaciones S1 y S4 son muy similares entre sí (con diferencias inferiores al 1\% en los resultados de las métricas) y respecto al modelo de referencia S8. Estas simulaciones tienen en común los factores accesibilidad y aptitud, lo que confirma una importante influencia de estos dos factores en el desarrollo de suelo urbano y una influencia de la vecindad menor de lo esperado.

Por otro lado, al comparar las simulaciones S1 y S15, se observa que el desarrollo de uso del suelo residencial es mayor en S1 (Mapa 2) y así lo confirman los valores del Cuadro 3, donde la métrica RPA para S1 supera a S15 en un 8\% aproximadamente. El desarrollo de uso de suelo productivo también varía, existiendo un desarrollo superior del 3\% en S15. Si bien las simulaciones S1 y S15 tienen en común los factores accesibilidad y vecindad, no se puede establecer la influencia directa de estos factores, ya que además se encuentran combinados con los factores aptitud, en el caso de S1, y aleatoriedad en S15.

Finalmente, las simulaciones S4 y S15 (con factor común la accesibilidad), arrojan un mayor ajuste y, por tanto, menor variación respecto al modelo de referencia en el desarrollo de suelo residencial en el primer caso, y de suelo productivo, en el segundo. Se observa que el factor accesibilidad, al combinarse con la aptitud (S4), desarrolla un uso residencial muy semejante al modelo de referencia (S8) con casi el valor RPA más elevado de todas las simulaciones (90,78\%), sólo superado en décimas por el S1. Aunque el porcentaje de acuerdo del S4 también es elevado para uso productivo ( $\mathrm{PPA}=94,09)$, se observa que la combinación con otros factores como la vecindad y la aleatoriedad (S15) no provoca casi variaciones de los resultados, registrándose en este caso el ajuste más elevado con el modelo de referencia (96,8\%). En cualquier caso, es destacable que, en líneas generales, la inclusión del factor de aleatoriedad provoca un ascenso del PPA y un descenso del RPA (ver S2 y S15, por ejemplo). 
Mapa 2. Resultados de las simulaciones de los usos de suelo residencial y productivo de los municipios Meco (columna izquierda), Azuqueca de Henares (columna central) y Los Santos de la Humosa (columna derecha). En la primera fila se representa el crecimiento real de suelo urbano registrado entre 2000 y 2010. S1 (N, A, S), S4 (A, S), S15 (N, A, R) Y S8 (N, A, S, R - modelo de referencia), siendo N: Vecindad; A: Accesibilidad; S: Aptitud; R: Aleatoriedad.
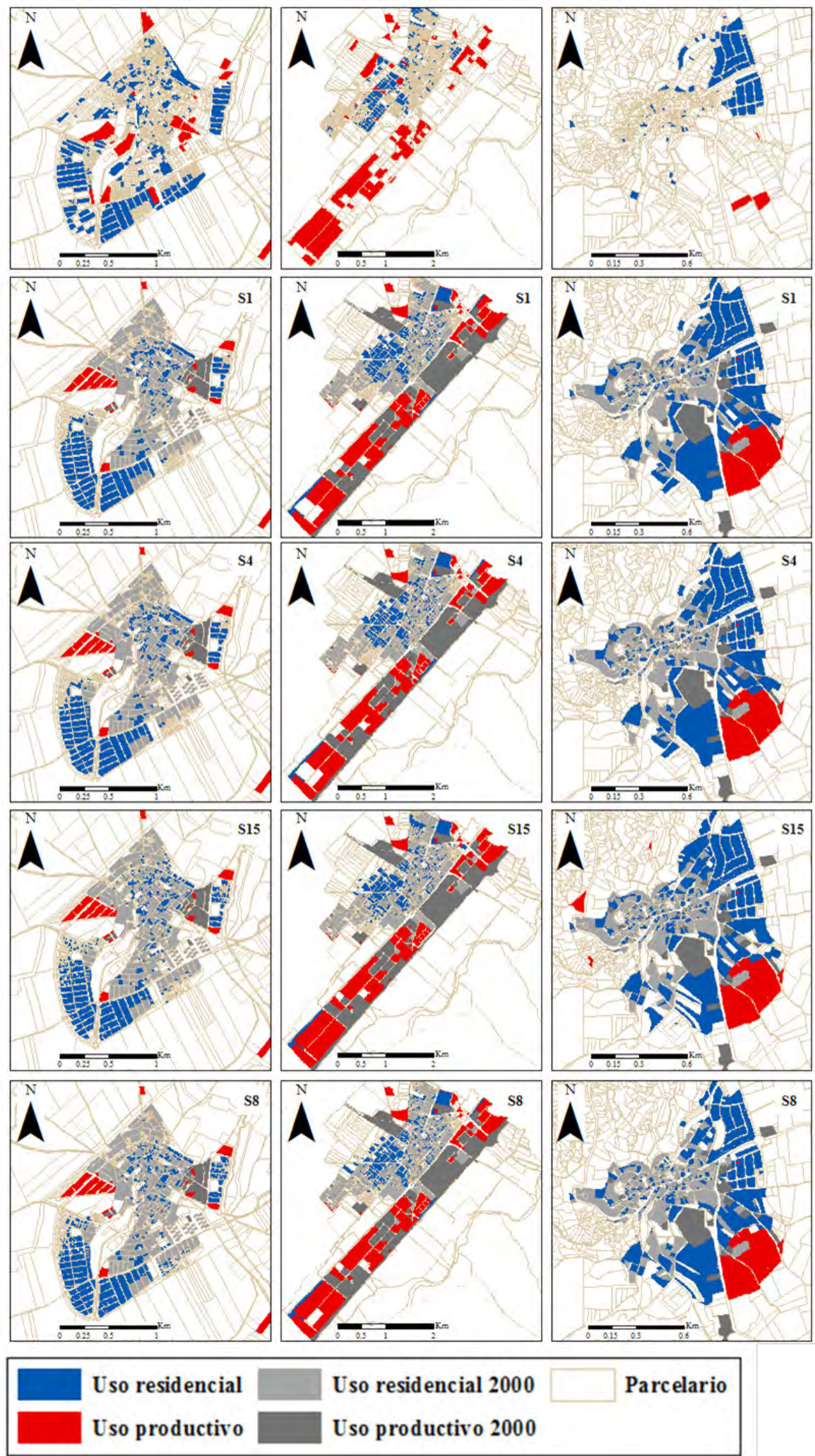

Fuente: Elaboración propia a partir de los datos de la Dirección General del Catastro (2013) y del Instituto Geográfico Nacional (2016). 
En líneas generales podemos decir que estos resultados estarían en consonancia con la especial configuración espacial del área de estudio, con un eje de máxima accesibilidad tan importante como es la A-2 y que supone un factor socioeconómico de desarrollo de primer orden en la zona. De hecho, una amplia mayoría de las zonas industriales se sitúan a lo largo de este eje en las simulaciones realizadas, condicionando esta vía el desarrollo urbanístico de la zona. Por otro lado, existe un crecimiento agregado en Meco y Los Santos de la Humosa, dada su configuración espacial.

\subsection{Comparación en función del número de parcelas y área desarrolladas}

El número de parcelas (a) y área desarrollada (b) para ambos usos urbanos en cada simulación, se encuentra representado en el Cuadro 4 de manera separada para evidenciar en detalle las diferencias en el desarrollo de parcelas tratándose de una estructura irregular. En primer lugar, observamos que, al igual que sucede con el modelo de referencia, el número de parcelas desarrolladas para suelo urbano suele ser mayor que el productivo, mientras que el área de las parcelas es mayor en el caso de suelo productivo que en el urbano. Este comportamiento se debe a que generalmente la geometría de las parcelas vacantes es muy diferente y con un nivel de fragmentación parcelaria más complejo, además del hecho de que las parcelas en principio clasificadas para uso productivo son, generalmente, de mayor tamaño, debido a la mayor demanda de superficie requerida para este uso.

Cuadro 4. Comparación de (a) número de parcelas y (b) área en hectáreas, de uso de suelo residencial y productivo, para las diferentes simulaciones respecto al modelo de referencia (S8).

(a)

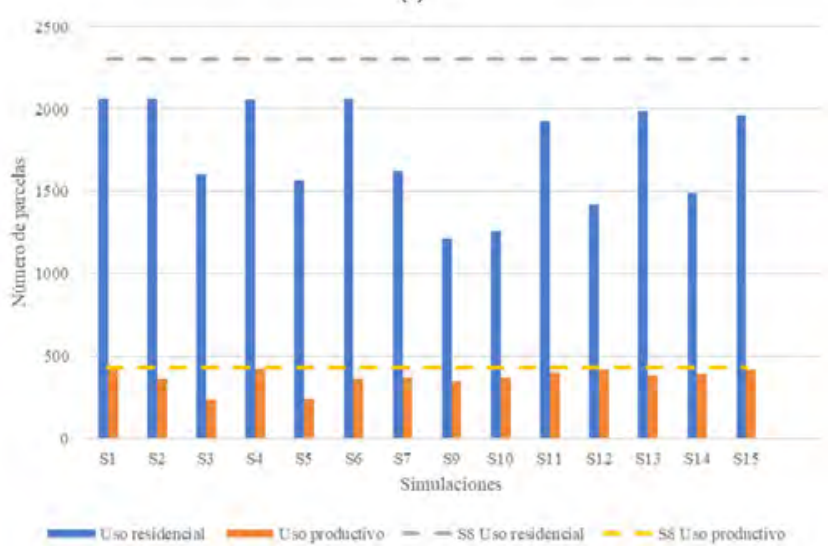

(b)

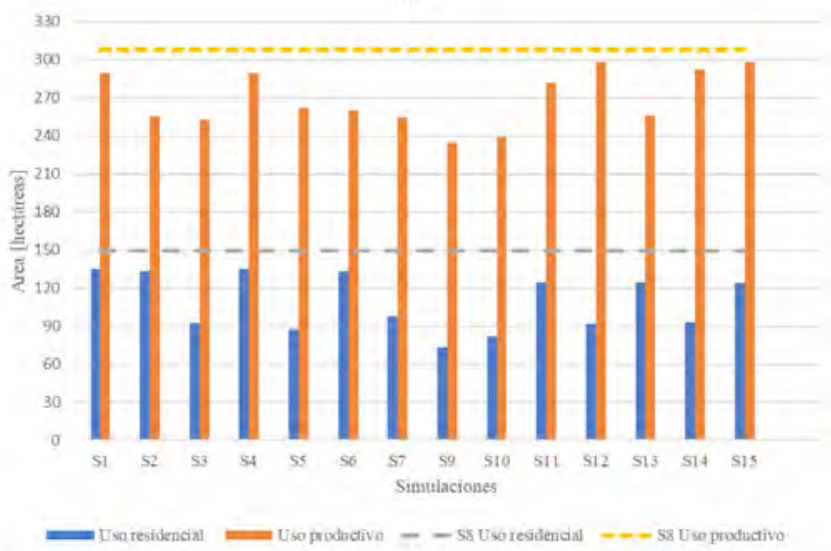


Con respecto a la influencia de los factores en las diferentes simulaciones, se observa que las simulaciones S1, S4 y S15 de nuevo son las más próximas al modelo de referencia para ambos tipos de desarrollo urbano (residencial y productivo), lo que corrobora la importante influencia de los factores accesibilidad y aptitud.

Al evaluar independientemente el desarrollo del uso residencial en número de parcelas (mayor a 1900 parcelas) y área (129 ha en promedio), las simulaciones S1, S2, S4, S6, S11, S13 son las más semejantes al modelo de referencia (con 2303 parcelas desarrolladas y una extensión promedio de 149 ha). Estas simulaciones tienen en común la inclusión del factor accesibilidad, lo que vuelve a confirmar que este factor es determinante para el desarrollo del uso residencial. Por otro lado, las simulaciones S12 (419 parcelas y 298 ha promedio), S14 (397 parcelas y 292 ha promedio), S15 (420 parcelas y 298 ha promedio) son las que mejor reproducen el desarrollo productivo con respecto a S8 (434 parcelas y 308 ha promedio), lo que significa que en este caso el factor aleatoriedad ejerce una influencia también significativa en el desarrollo de este tipo de suelo.

Las simulaciones S3, S5, S7, S9, S10 presentan una semejanza global que varía entre el 55\% y el $85 \%$, porcentajes que, para ser comparados con el modelo de referencia, han sido evaluados independientemente por área y número de parcelas.

\subsection{Resultados del análisis de sensibilidad (efectos principal y total)}

Utilizando el método de la descomposición de la varianza, podemos determinar la contribución concreta de cada uno de los factores del modelo y en conjunto a la variación de los resultados del mismo, complementando y matizando, por tanto, los resultados que nos ofrecía el cálculo de las distintas métricas incluidas en el Cuadro 3. Así, como puede comprobarse en los índices de primer orden que aparecen en el Cuadro 5, el factor que contribuye en mayor medida al desarrollo urbano del modelo MUGICA es la accesibilidad, con una influencia de 0,606. Analizando el desarrollo del suelo residencial independientemente, este aspecto se confirma, elevándose hasta un 0,894 su influencia, seguida muy de lejos por la aptitud que representa apenas un 0,037 . Por otro lado, el desarrollo de uso de suelo productivo se ve influenciado de manera más evidente (pero sin alcanzar valores tan altos), tanto por la aptitud, como por la accesibilidad (0,178 y 0,169 respectivamente).

Si observamos los datos de las sumas de los índices de primer orden comprobamos que, en el caso de suelo productivo, se encuentra por debajo de $0,6(0,43)$, lo que está indicando que una parte importante de la variación del resultado vendría explicada por la interacción entre los distintos factores del modelo (hasta un 57\%) y no por alguno de ellos individualmente, como sí sucede en el desarrollo de suelo residencial. En todos los casos, tanto la vecindad como la aleatoriedad presentan valores bastante menos importantes, lo que nos está indicando que, de manera individual, estos son los factores que menos están influyendo en la variación de los resultados.

Si observamos los índices de segundo orden y sucesivos, comprobamos que para que haya una influencia realmente importante en los resultados, tiene que darse una interacción de hasta tres de los factores incluidos en el modelo, explicando la combinación de la accesibilidad, la aleatoriedad y la aptitud la mayor parte de la varianza de los resultados en cualquier tipo de desarrollo de suelo urbano. La interactuación de la vecindad, la aptitud y la aleatoriedad, sin embargo, es la que menos variaciones produce en los resultados del modelo. Especialmente en el suelo residencial, comprobamos cómo es la combinación de la accesibilidad con cualquiera de los otros factores la 
que está marcando la diferencia en los resultados. La interpretación de estos resultados es que, aunque algunos de los factores de manera individual no aportan mucho a la varianza de salida, no podríamos prescindir de ellos porque juegan un papel importante interactuando con los restantes. Pero, por otro lado, si sustituyésemos todos los factores, menos la accesibilidad, por un valor constante, probablemente obtendríamos prácticamente los mismos resultados.

Cuadro 5. Índices de sensibilidad de primer orden $\eta_{\mathrm{i}}$, segundo orden $\eta_{\mathrm{ij}}$, tercer orden $\eta_{\mathrm{ijk}} \mathrm{y}$ total $\eta_{\mathrm{T}}$ de los factores de vecindad $(N)$, accesibilidad $(A)$, aptitud $(S)$ y aleatoriedad $(R)$ para las parcelas desarrolladas a uso residencial o productivo.

\begin{tabular}{|c|c|c|c|}
\hline \multirow{2}{*}{ Índices } & \multicolumn{3}{|c|}{ Usos de suelo } \\
\hline & Residencial & Productivo & Urbano \\
\hline$\eta_{s}$ & 0,037 & 0,178 & 0,12 \\
\hline$\eta_{N}$ & 0,005 & 0,031 & 0,019 \\
\hline$\eta_{\mathrm{A}}$ & 0,894 & 0,169 & 0,606 \\
\hline$\eta_{R}$ & 0,014 & 0,053 & 0,004 \\
\hline$\sum \eta_{i}$ & 0,950 & 0,430 & 0,749 \\
\hline$\eta_{N A}$ & 0,004 & 0,036 & 0,02 \\
\hline$\eta_{\mathrm{NS}}$ & 0,002 & 0,006 & 0,005 \\
\hline$\eta_{N R}$ & 0,013 & 0,041 & 0,032 \\
\hline$\eta_{\mathrm{AS}}$ & 0,001 & 0,021 & 0,01 \\
\hline$\eta_{A R}$ & 0,002 & 0,002 & 0,002 \\
\hline$\eta_{S R}$ & 0,012 & 0,022 & 0,021 \\
\hline$\eta_{\text {NAS }}$ & 0,944 & 0,399 & 0,763 \\
\hline$\eta_{\mathrm{NAR}}$ & 0,913 & 0,296 & 0,645 \\
\hline$\eta_{\text {NSR }}$ & 0,058 & 0,266 & 0,147 \\
\hline$\eta_{\text {ASR }}$ & 0,945 & 0,760 & 0,842 \\
\hline$\eta_{\mathrm{TS}}$ & 1,999 & 1,651 & 1,908 \\
\hline$\eta_{\mathrm{TN}}$ & 1,939 & 1,075 & 1,631 \\
\hline$\eta_{\mathrm{TA}}$ & 3,703 & 1,683 & 2,888 \\
\hline$\eta_{T R}$ & 1,957 & 1,440 & 1,693 \\
\hline
\end{tabular}

En cuanto a los índices de sensibilidad totales, encontramos que la accesibilidad vuelve a dominar en todos los tipos de suelo, lo que reforzaría la idea anterior de que el resto de los factores podría ser sustituido por un valor constante. Sin embargo, en el caso del suelo productivo, la diferencia no es tan importante con el valor obtenido para el factor aptitud, lo que corrobora las deducciones realizadas sobre el efecto de estos dos factores en los índices de primer, segundo y tercer orden.

\subsection{Mapa de frecuencias}

En el Mapa 3 aparecen cartografiadas las parcelas que con mayor frecuencia se desarrollaron para uso de suelo urbano en las distintas simulaciones realizadas. Observando la distribución espacial de las parcelas y las características del territorio analizado (Mapa 4), para ambos suelos 
en conjunto (residencial y productivo), la mayor contribución a la frecuencia de desarrollo vendría dada por el factor accesibilidad, seguida del factor aptitud y en menor medida, pero también importante, el factor vecindad, ligado al uso del suelo pre-existente para lograr la ubicación del desarrollo con mayor exactitud en la calibración. La alta frecuencia en la selección de parcelas para desarrollo de suelo residencial se explica de manera más importante por la contribución del factor accesibilidad, debido a la proximidad de estas a las principales vías de comunicación. En el caso del uso productivo, los factores que contribuyen mayoritariamente son la accesibilidad y la aptitud, dadas las características comunes (clasificación del suelo, distancia a la red hidrográfica y pendiente) en la mayoría de las parcelas vacantes de cada uno de los tres municipios.

Mapa 3. Mapas de frecuencia para usos del suelo residencial y productivo de los municipios (a) Meco, (b) Azuqueca de Henares, (c) Los Santos de la Humosa.

Uso de suelo residencial Uso de suelo productivo
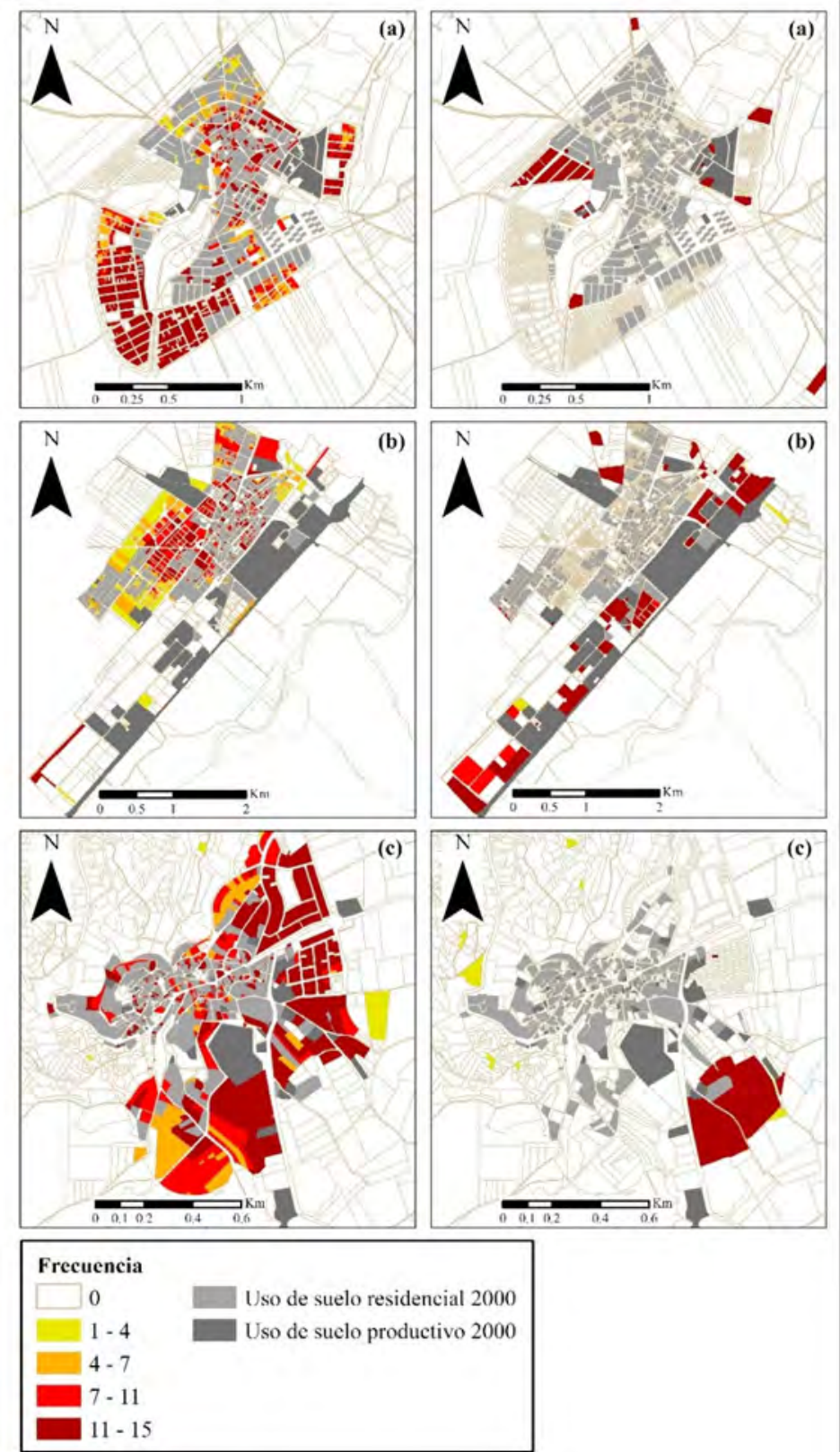

Fuente: Elaboración propia a partir de los datos de la Dirección General del Catastro (2013) y del Instituto Geográfico Nacional (2016). 
La frecuencia del desarrollo de suelo urbano atribuida al factor aptitud es determinante cuando las diferencias topográficas son abruptas. Tal es el caso presentado en el municipio de Los Santos de La Humosa (c), donde la concentración de parcelas susceptibles de ser desarrolladas como uso productivo se produce en el sector sur, influenciado por la baja pendiente en esta zona, condición más adecuada para desarrollar este tipo de suelo.

Mapa 4. Representación de la red vial, red hidrográfica y pendiente de los municipios de Meco, Azuqueca de Henares y Los Santos de la Humosa.

Meco

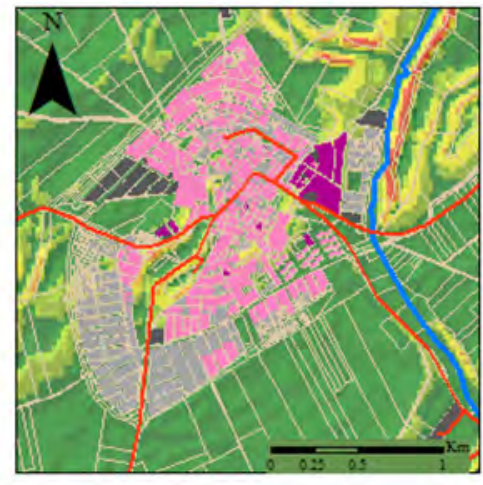

Azuqueca de Henares Los Santos de la Humosa
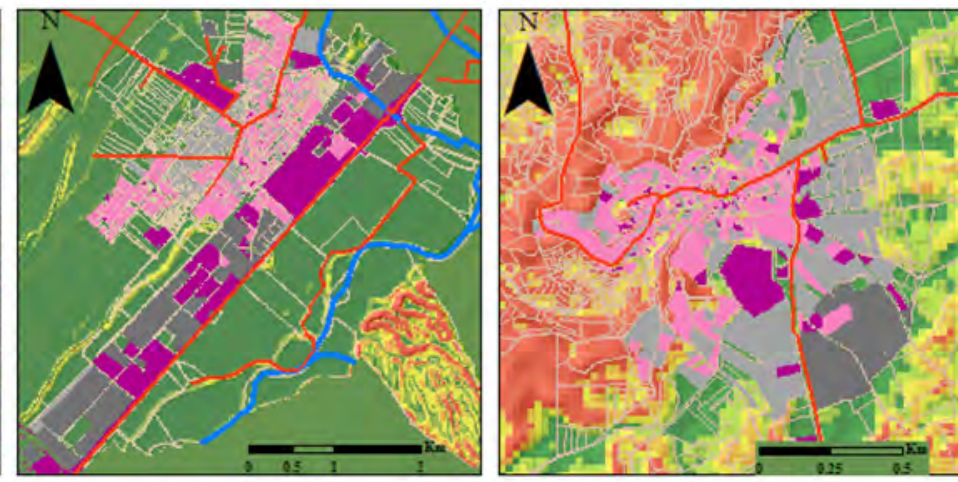

Pendiente $\%$

0.5

$\square 15-20 \longrightarrow$ Vias

Uso de suelo residencial \$s

Uso de suelo productivo $S S$

Uso de suelo residencial 2000 Parcelario

Uso de suelo productivo 2000

$10-15$

Fuente: Elaboración propia a partir de los datos de la Dirección General del Catastro (2013) y del Instituto Geográfico Nacional (2016).

\section{Discusión y conclusiones}

El presente trabajo es una primera contribución a la aplicación de un AS a un modelo de desarrollo urbano basado en AC irregulares. Para ello, se programó de forma paralela al modelo las rutinas necesarias para llevar a cabo la validación del mismo. El principal objetivo que perseguía este trabajo era emplear el AS para determinar la influencia de los factores del modelo MUGICA, en el que se asume que cada uno de los factores implementados en el modelo podría presentar una influencia diferente sobre los resultados generados. Los resultados muestran que este tipo de AS, adaptación y combinación de los métodos globales y de tipo OAT aplicados en el campo del modelado numérico, son aplicables de manera sencilla para este tipo de modelos, en los que los resultados no son cuantitativos, sino una variable de tipo binario (urbanizado o no urbanizado).

Por otro lado, al realizarse en el período de calibración del modelo, es posible complementar su validación en simulaciones prospectivas, pues, como es sabido, el proceso de calibración sólo nos asegura que el modelo es capaz de reproducir a futuro las condiciones experimentadas en el pasado, es decir, simular un escenario tendencial, pero no puede presuponerse validado para explorar otro tipo de escenarios futuros posibles o deseables, que no necesariamente tienen que coincidir con lo ocurrido en el pasado (Barreira-González et al., 2015a). De esta manera, se ha podido comprobar la correcta implementación de los factores (es decir, que el modelo está correctamente construido), además de garantizar la consistencia y continuidad del modelo en el tiempo. 
En este punto hemos de mencionar que, en el trabajo cuya metodología se ha pretendido reproducir (Barreira-González et al., 2015a), se pudo comprobar cómo, a pesar de tratarse de un modelo calibrado, el AS mostró que un factor tan determinante como la accesibilidad en la expansión urbana, y supuestamente determinante en el modelo desarrollado, no mostraba apenas influencia en la variación de los resultados, con lo que no quedaba asegurado que el modelo estuviese correctamente construido para el escenario de futuro que se pretendía simular (de innovación y sostenibilidad, y no tendencial). Probablemente en este caso particular la accesibilidad sí ha mostrado tener la influencia que se presuponía, porque el área de estudio es mucho más pequeña y condicionada por una vía tan importante como la A-2, sin embargo, en el trabajo que venimos mencionando, se realizó una simulación para toda la región de Madrid, lo que pudo de alguna manera diluir el efecto de este factor.

En cualquier caso, se demuestra que tan sólo el proceso de calibración no es suficiente para concluir que se ha desarrollado el modelo adecuado, siendo importante combinar procesos de calibración y de validación, especialmente para constatar la viabilidad y garantizar la correcta utilización del mismo en la realización de simulaciones a futuro, sin datos reales con los que poder contrastar los resultados. Pensamos que este tipo de análisis contribuyen, por tanto, a robustecer el uso de estos modelos basados en AC tan ampliamente utilizados en este campo.

Adicionalmente, se ha podido demostrar que es claramente insuficiente realizar cualquier tipo de AS que se ciña exclusivamente a alguno de los factores del modelo, no teniendo en cuenta el efecto de cada uno de ellos, tanto de manera individual como en su interactuación con los restantes. Así, los ejemplos que encontramos en la literatura (Kocabas y Dragicevic, 2006; Dahal y Chow, 2015; Abolhasani et al., 2016; entre otros), centrados exclusivamente en el efecto de la vecindad (tanto en modelos de base espacial regular como irregular), no estarían validando los resultados del modelo desarrollado de forma completa, cuestión absolutamente necesaria para poder garantizar unos resultados que se pretenden utilizar de manera prospectiva.

Finalmente, al analizar los resultados del AS para MUGICA, se identificó y ratificó que, como era de esperar, los factores accesibilidad y aptitud son los más susceptibles a cualquier variación en el modelo, debido a las diferentes características que cada uno de estos factores presentan en los municipios sobre los que se implementó este. Por otro lado, vecindad y aleatoriedad son factores complementarios cuyos efectos principales favorecen la precisión del desarrollo urbano de MUGICA. Lo anterior confirma la correcta construcción del modelo de partida y su capacidad de reproducir los principios clásicos basados en AC, en un entorno irregular, utilizando parcelas catastrales y para otro tipo de simulaciones a futuro que no sean estrictamente tendenciales.

Adicionalmente, la elaboración de los mapas de frecuencias puede resultar de gran ayuda en las tareas de planificación, contribuyendo a identificar aquellas zonas con mayor vocación para ser convertidas en uso residencial o productivo, por tratarse de los resultados más robustos y repetidos, independientemente de las modificaciones que se realicen en los parámetros del modelo.

Pensamos, en definitiva, que este trabajo puede servir de base para aplicar la metodología a otros casos que empleen modelos basados en AC irregulares en este y otros tipos de cambios de usos del suelo. En especial para validar parcialmente simulaciones de escenarios futuros de desarrollo de uso de suelo urbano basados en estructuras irregulares, más apropiados para simular con más realismo un fenómeno de este tipo y a escala municipal o de ciudad, donde la utilización del píxel pierde su interés por ser una representación excesivamente sencilla y generalista del territorio (Pinto y Antunes, 2010; Zelaya et al., 2016). Si bien en el pasado la utilización de la celda facilita- 
ba cálculos matemáticos y minimizaba el tiempo de ejecución de estos modelos, los avances en computación han demostrado que esta ya no es una excusa razonable, especialmente para aplicaciones a escala de detalle, como se ha demostrado en el desarrollo de MUGICA y en la validación aquí presentada.

\section{Agradecimientos}

Este trabajo se ha realizado dentro de las actividades del proyecto de investigación coordinado SIMURBAN2: Instrumentos de Geosimulación y planificación ambiental en la ordenación territorial de ámbitos metropolitanos. Aplicación a escalas intermedias, financiado por el Ministerio de Economía y Competitividad (Ref: CSO2012-38158-C02-01).

\section{Referencias bibliográficas}

- Abolhasani, S.; Taleai, M.; Karimi, M. y Rezaee Node, A. (2016). «Simulating urban growth under planning policies through parcel-based cellular automata (ParCA) model». International Journal of Geographical Information Science, 30(11), 2276-2301.

- Aguilera, F.; Valenzuela, L.M. y Bosque, J. (2010). «Simulación de escenarios futuros en la aglomeración urbana de Granada a través de modelos basados en autómatas celulares». Boletín de la Asociación de Geógrafos Españoles, 54, 271-300.

- Antrop, M. (2004). «Landscape change and the urbanization process in Europe». Landscape and Urban Planning, 67 (1-4), 9-26.

- Barredo, J.; Demichelli, L.; Kasanko, M. y McComick, N. (2004). «Modelling Future urban scenarios in developing countries: An application case study in Lagos, Nigeria». Environment and Planning B: Planning and Design, 32, 65-84.

- Barredo, J.I. y Gómez-Delgado, M. (2008). «Towards a set of IPCC SRES urban land use scenarios: modelling urban land use in the Madrid region». En: Paegelow, M. y Camacho M.T. (Eds). Modelling Environmental Dynamics. Berlin: SpringerHeidelberg, 363-385.

- Barreira-González, P.; Aguilera-Benavente, F. y Gómez-Delgado, M. (2015a). «Partial validation of cellular automata based model simulations of urban growth: An approach to assessing factor influence using spatial methods». Environmental Modelling \& Software, 69, 77-89.

- Barreira-González, P.; Gómez-Delgado, M. y Aguilera-Benavente, F. (2015b). «From raster to vector cellular automata models: A new approach to simulate urban growth with the help of graph theory». Computers, Environment and Urban Systems, 35, 119-131.

- Barreira-González, P. y Barros, J. (2016). «Configuring the neighbourhood effect in irregular cellular automata based models». International Journal of Geographical Information Science, 31(3), 617-636.

- Barreira-González, P.; Aguilera-Benavente, F. y Gómez-Delgado, M. (2017). «Implementation and calibration of a new irregular cellular automata-based model for local urban growth simulation: The MUGICA model». Environment and Planning B: Urban Analytics and City Science, publicado on-line (DOI: 10.1177/2399808317709280).

- Batty, M. y Xie, Y. (1994). «From Cells to Cities». Environment and Planning B: Urban Analytics and City Science, 21, 31-48.

- Batty, M. y Xie, Y. (1997). «Possible Urban Automata». Environment and Planning B: Urban Analytics and City Science, 24, 175 - 192.

- Batty, M.; Xie, Y. y Sun, Z. (1999). «Modeling urban dynamics through GIS-based cellular». Computers Environment and Urban Systems, 23, 205-233.

- Berberoglu, S.; Akin, A. yClarke, K. (2016). «Cellular automata modeling approaches to forecast urban growth for Adana, Turkey: a comparative approach». Landscape and Urban Planning, 153, 11-27.

- Brown, D.; Scott P.; Rick R.; Moira Z. y William R. (2005). «Path dependence and the validation of agent-based spatial models of land use». International Journal of Geographical Information Science, 19(2), 153-174. 
- Cantergiani, C.C. y Gómez-Delgado, M. (2016). «Diseño de un modelo basado en agentes para simular el crecimiento urbano en el Corredor del Henares (Comunidad de Madrid)». Boletín de la Asociación de Geógrafos Españoles, 70, 259-283.

- Cantergiani, C.C. y Gómez-Delgado, M. (2018). «Urban land allocation model of territorial expansion by urban planners and housing developers». Environments, 5, 5. https://doi.org/10.3390/environments5010005

- Cao, M.; Bennett, S.J.; Shen, Q. y Xu, R. (2016). «A bat-inspired approach to define transition rules for a cellular automaton model used to simulate urban expansion». International Journal of Geographical Information Science, 30(10), 1961-1979.

- Chuvieco, E. (2010). Teledetección Ambiental: La observación de la Tierra desde el Espacio. 4a edición. Barcelona: Ariel Ciencia.

- Crosetto, M. y Tarantola, S. (2001). «Uncertainty and sensitivity analysis: tools for GIS-based model implementation». International Journal of Geographical Information Science, 15(5), 415-437.

- Dahal, K.R. y Chow, T.E. (2014). «An agent-integrated irregular automata model of urban land-use dynamics». International Journal of Geographical Information Science, 28(11), 2281-2303.

- Dahal, K.R. y Chow, T.E. (2015). «Characterization of neighborhood sensitivity of an irregular cellular automata model of urban growth». International Journal of Geographical Information Science, 29(3), 475-497.

- de Noronha, T. y Vaz, E. (2015). «Framing urban habitats: The small and medium towns in the peripheries». Habitat International, 45, 147-155.

- Díaz-Pacheco, J. y Hewitt, R. (2014). «Modelado de cambios de usos de suelo urbano a través de redes neuronales artificiales. Comparación con dos aplicaciones de software». GeoFocus. Revista Internacional de Ciencia y Tecnología de la Información Geográfica, 14, 1-22.

- Engelen, G.; Geertman S.; Smits P. y Wessels C. (1999). «Dynamic GIS and Strategic Physical Planning Support: A Practical Application». En: Stillwell J.; Geertman S.; Openshaw S. (Eds): Geographical Information and Planning. Advances in Spatial Science (The Regional Science Series). Berlin: Springer Heidelberg, 87-111.

- Engelen, G. y White, R. (2008). «Validating and Calibrating Integrated Cellular Automata Based Models of Land Use Change». En: Albeverio, S; Andrey, D.; Giordano, P. y Vancheri A. (Eds.). The Dynamics of Complex Urban Systems. Switzerland: Springer, 185-211.

- Fahmi, F. Z.; Hudalah, D.; Rahayu, P. y Woltjer, J. (2014). «Extended urbanization in small and medium-sized cities: The case of Cirebon, Indonesia». Habitat International, 42, 1-10.

- Gómez-Delgado, M. y Bosque-Sendra, J. (2004). «Sensitivity analysis in multicriteria spatial decision making: a review». Human Ecological Risk Assessment, 10(6), 1173-1187.

- Gómez-Delgado, M. y Tarantola, S. (2006). «GLOBAL sensitivity analysis, GIS and multicriteria evaluation for a sustainable planning of a hazardous waste disposal site in Spain». International Journalof Geographic Information Science, 20(4), 449-466.

- Hansen, H. S. (2010). «Modelling the future coastal zone urban development as implied by the IPCC SRES and assessing the impact from sea level rise». Landscape and Urban Planning, 98,141-149.

- Hewitt, R. y Escobar Martínez, F. (2011). «The territorial dynamics of fast-growing regions: Unsustainable land use change and future policy challenges in Madrid, Spain». Applied Geography, 31, 650-667.

- Hewitt, R. y Díaz Pacheco, J. (2017). «Stable models for metastable systems? Lessons from sensitivity analysis of a Cellular Automata urban land use model». Environmental Modelling \& Software, 62, 113-124.

- Instituto Nacional de Estadística, INE, (2017): www.ine.es/dyngs/INEbase/es/categoria.htm?c=Estadistica_P\&c id=1254735572981 [consulta: 20 de julio de 2017]

- Kalnay, E. y Cai M. (2003). «Impact of urbanization and land-use change on climate». Nature, 423, 528-531.

- Kocabas, V. y Dragicevic, S. (2006) «Assessing a cellular automata model behavior using a sensitivity analysis approach». Computers Environmentand Urban Systems,30, 921-953.

- Lilburne, L. y Tarantola, S. (2009). «Sensitivity analysis of spatial models». International Journal of Geographic Information Science, 23(2), 151-168.

- Liu, Y.; He Q.; Tan, R.; Liu, Y. y Yin, C. (2016). «Modeling different urban growth patterns based on the evolution of urban form: A case study from Huangpi, Central China». Applied Geography, 66, 109-118.

- Lu, Y.; Cao, M; y Zhang, L. (2015). «A vector-based Cellular Automata model for simulating urban land use change». Chinese Geographical Science, 25(1), 74-84. 
- Moreno, N.; Ménard, A. y Marceau, D. J. (2008). «VecGCA: a vector-based geographic cellular automata model allowing geometric transformations of objects». Environment and Planning B-Planning \& Design, 35, 647-665.

- Muller, K.; Steinmeier, C. y Kuchler, M. (2010). «Urban growth along motorways in Switzerland». Landscape and Urban Planning, 98, 3-12.

- Olazabal, E. y Bellet, C. (2018). «Procesos de urbanización y artificialización del suelo en las aglomeraciones urbanas españolas (1987-2011)». Cuadernos Geográficos, 57(2), 189-210.

- Open Source Geospatial Foundation. 2000. Geospatial Data Abstraction Library (GDAL).

- Paegelow, M. y Camacho, M. (2008). Modelling environmental dynamics. Berlin: Springer Verlag.

- Pinto, N.N. y Antunes, A.P. (2010). «A cellular automata model based on irregular cells: application to small urban areas «. Environment and Planning B: Planning and Design, 37(6), 1095-1114.

- Plata Rocha, W.; Gómez-Delgado, M. y Bosque Sendra, J. (2009). Cambios de usos del suelo y expansión urbana en la comunidad de Madrid (1990-2000). Scripta-Nova, 13, 283-309.

- Plata-Rocha, W.; Gómez-Delgado, M. y Bosque-Sendra, J. (2011): «Simulating urban growth scenarios using GIS and Multicriteria Evaluation techniques. Case study: Madrid Region, Spain», Environment and Planning B. Planning and design, 38 (6), 1012-1031.

- Plata-Rocha, W.; Gómez-Delgado, M. y Bosque-Sendra, J. (2012). «Proposal for the introduction of the spatial perspective in the application of global sensitivity analysis». Journal of Geographical Information Systems, 4, 503513.

- Saltelli, A.; Chan, K. y Scott, E., 2000. Sensitivity Analysis. New York: Wiley.

- Salvati, L. y Sabbi, A. (2011). «Exploring long-term land cover changes in an urban region of southern Europe». International Journal of Sustainable Development and World Ecology, 18, 273-282.

- Santé, I.; García, A.; Miranda, D. y Crecente, R. (2010). «Cellular automata models for the simulation of real world urban processes: A review and analysis». Landscape and Urban Planning, 96, 108-122.

- Saraiva, M. y Pinho, P. (2017). «Spatial modelling of commercial spaces in medium-sized cities». Geojournal, 82, 433-454.

- Semboloni, F. (1997). «An urban and regional model based on cellular automata». Environment and Planning B: Urban Analytics and City Science, 24, 589-612.

- Stevens, D.; Dragicevic, S. y Rothley, K. (2006). «iCity: A GIS-CA modelling tool for urban planning and decision making». Environmental Modelling and Software, 22, 761-773.

- Stevens, D. y Dragicevic, S. (2007). «A GIS-based irregular cellular automata model of land-use change». Environment and Planning B: Planning and Design, 34(4), 708-724.

- Triantakonstantis, D. y Mountrakis, G. (2012). «Urban growth prediction: a review of computational models and human perceptions». Journal of Geographic Information System, 4, 555-587.

- Urgilez Clavijo, A.; Barreira González, P. y Gómez Delgado, M. (2018). «Análisis de sensibilidad aplicado a modelos de crecimiento urbano basado en autómatas celulares de estructura irregular».TIG: perspectivas multidisciplinares en la sociedad del conocimiento. Actas del XVIII Congreso Nacional de TIG. Valencia: Universidad de Valencia, 568-579.

- Van Vliet, J.; Bregt, A.K.; Brown, D.G.;Van Delden, H.; Heckbert, S. y Verburg, P.H. (2016). «A review of current calibration and validation practices in land-change modeling». Environmental Modelling \& Software, 82, $174-182$.

- Verburg, P.; Schot, P.; Djist, M. J. y Veldkamp, A. (2004). «Land Use change modeling: current practice and research priorities. GeoJournal, 61, 309-324.

- White, R.; Engelen, G. y Uljee, I. (1997). «The use of constrained cellular automata for high-resolution modelling of urban land-use dynamics». Environmentand Planning B: Planning and Design, 24, 323-343.

- Wolfram, S. (1984). «Cellular Automata as Models of Complexity». Nature, 311 (5985), 419-424.

- Xu, E. y Zhang, H. (2013). «Spatially-explicit sensitivity analysis for land suitability evaluation». Applied Geography, 45, 1-9.

- Zelaya, K.; Van Vliet, J. y Verburg, P.H. (2016). «Characterization and analysis of farm system changes in the Mar Chiquita basin, Argentina». Applied Geography, 68, 95-103. 


\section{Sobre las/os autoras/es}

Andrea Urgilez Clavijo

Ingeniera de Sistemas por la Universidad del Azuay (Cuenca, Ecuador), MSc en Tecnologías de la Información Geográfica por la Universidad de Alcalá (España). Actualmente, Docente-Investigadora en la Universidad del Azuay. Investigadora pre doctoral en el Departamento de Geografía y Ordenación del Territorio de la Universidad de Zaragoza.

Montserrat Gómez Delgado

Doctora en Geografía por la Universidad de Alcalá en 2003 y profesora Titular de Geografía Humana en el Departamento de Geología, Geografía y Medio Ambiente de la Universidad de Alcalá. Su historial docente e investigador está estrechamente relacionado con la utilización de las Tecnologías de la Información Geográfica en diversos campos como la localización óptima de instalaciones no deseables, cartografía de riesgos, asignación óptima de usos del suelo y la simulación del crecimiento urbano a partir de distintos modelos (técnicas EMC, autómatas celulares, basados en agentes).

Pablo Barreira GonzÁlez

Pablo Barreira González, es PhD y MSc en Tecnologías de la Información Geográfica e Ingeniero en Geodesia y Cartografía por la Universidad de Alcalá. Sus líneas de investigación se han centrado en la modelización de sistemas complejos tales como el crecimiento urbano a través de autómatas celulares empleando estructuras irregulares. Dentro de las mismas ha abordado diferentes etapas del proceso de modelización como es la calibración, la validación a través de análisis de sensibilidad o la determinación de diferentes métodos cuantitativos y cualitativos de comparación de simulaciones. 